\title{
Hemangioma of the cervical esophagus: A rare case of dysphagia mimicking a large esophageal polyp on endoscopy
}

\author{
Juan A. Santamaria-Barria, MD, ${ }^{\mathrm{a}}$ Farzaneh Banki, MD, ${ }^{\mathrm{a}, \mathrm{b}}$ Suresh Rajendran, $\mathrm{MD},{ }^{\mathrm{b}}$ and \\ Craig Floyd, MD, ${ }^{\mathrm{b}}$ Houston, Tex

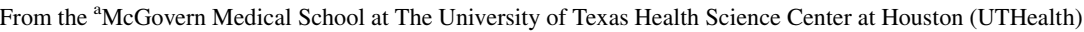 \\ and ${ }^{\mathrm{b}}$ Memorial Hermann Southeast Esophageal Disease Center, Houston, Tex. \\ Disclosures: Authors have nothing to disclose with regard to commercial support. \\ Received for publication March 2, 2016; revisions received April 12, 2016; accepted for publication April 17 \\ 2016; available ahead of print May 24, 2016 \\ Address for reprints: Farzaneh Banki, MD, Department of Cardiothoracic and Vascular Surgery, McGovern Med- \\ ical School at UTHealth, Esophageal Disease Center, Memorial Hermann Southeast, 11800 Astoria Blvd, \\ Houston, TX 77089 (E-mail: Farzaneh.Banki@uth.tmc.edu). \\ J Thorac Cardiovasc Surg 2016;152:e59-61 \\ $0022-5223 / \$ 36.00$ \\ Copyright (C) 2016 by The American Association for Thoracic Surgery \\ http://dx.doi.org/10.1016/j.jtcvs.2016.04.050
}

Esophageal benign soft tissue neoplasms are rare, with the most common being leiomyoma. Hemangioma of the esophagus is a rare entity, with few cases reported. ${ }^{2}$ We present a rare case of dysphagia in a patient with hemangioma of the cervical esophagus mimicking a large esophageal polyp on endoscopy.

\section{CLINICAL SUMMARY}

The patient was a 67 -year-old woman with a 10 -year history of cervical dysphagia to solids. This had progressed

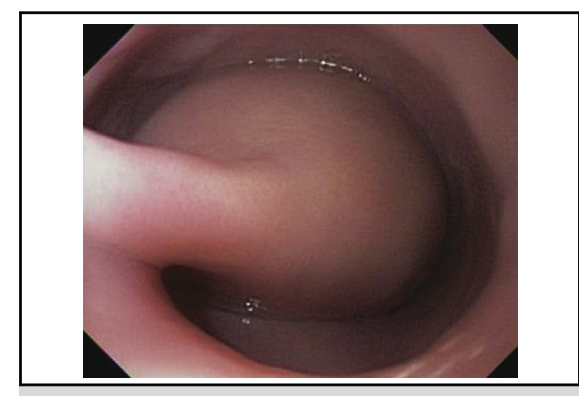

Endoscopic view showing a nearly obstructing cervical esophageal submucosal mass.

\section{Central Message}

Report stresses the need for comprehensive evaluation of dysphagia by presenting the misleading diagnosis of a large polyp, which was corrected by obtaining videoesophagogram and CT scan of the neck.

See Editorial Commentary page e63. during the last year to dysphagia to liquids, associated with a choking sensation with both solids and liquids.
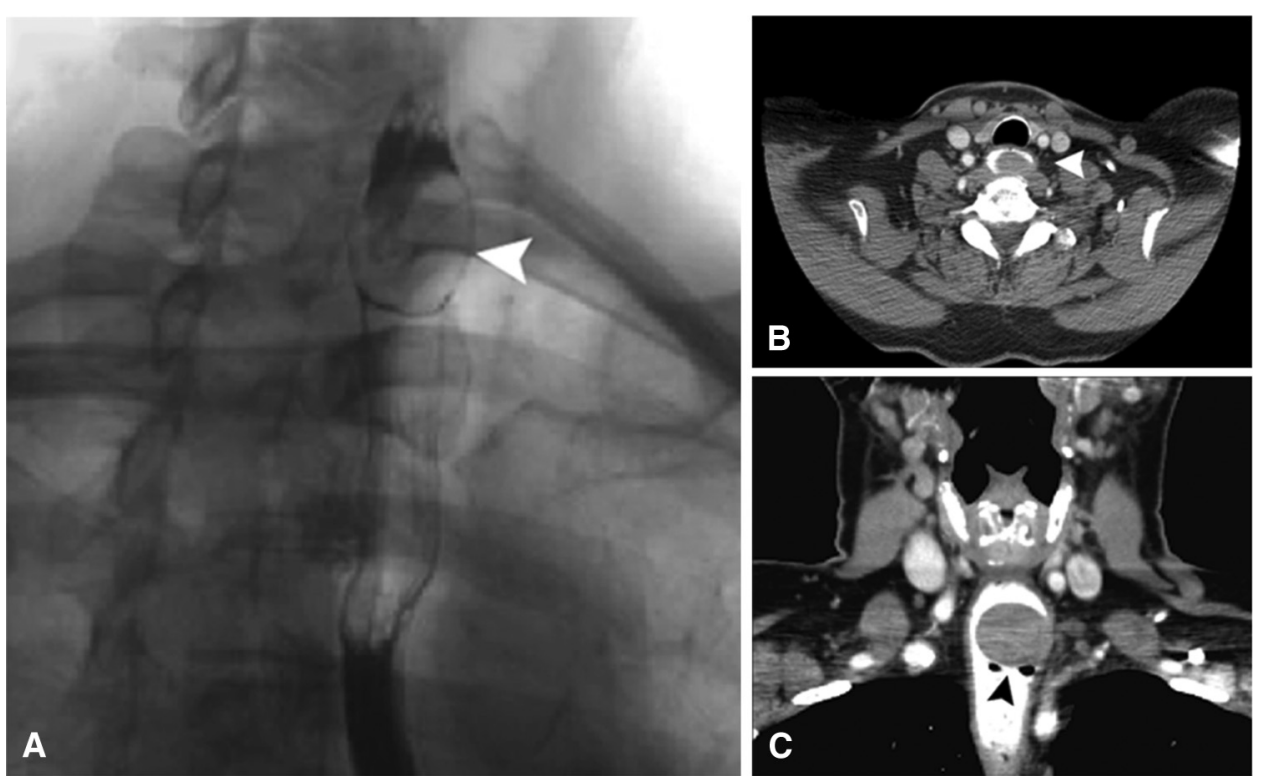

FIGURE 1. A, Videoesophagram showing an oval mass arising from the cervical esophageal wall with pool of barium above the esophageal mass (arrow). $\mathrm{B}$, Neck computed tomographic axial views showing a 2.5 -cm mass arising from the cervical esophageal wall. C, Neck computed tomographic coronal views showing a $2.5-\mathrm{cm}$ mass (arrow) arising from the cervical esophageal wall. 


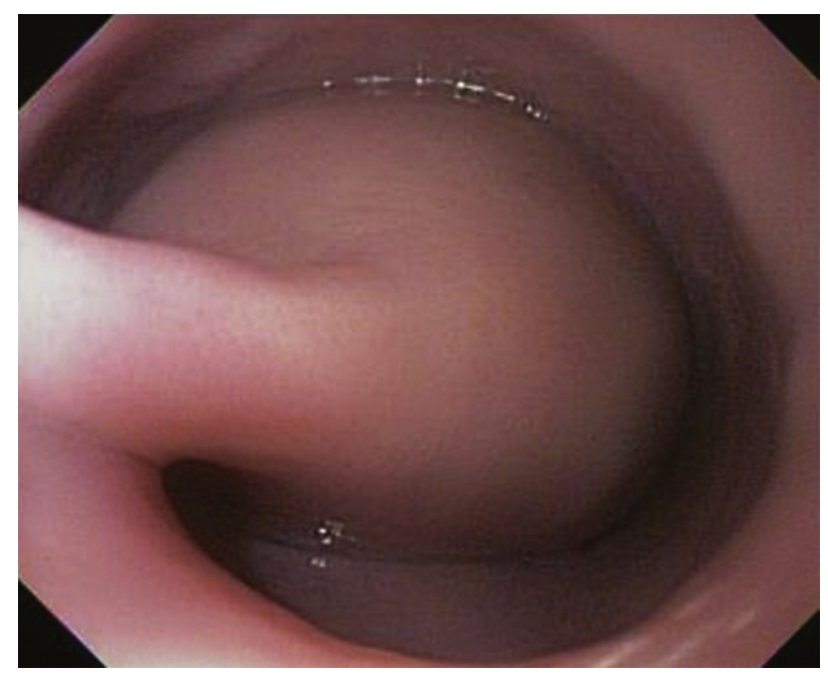

FIGURE 2. Endoscopic view showing a nearly obstructing cervical esophageal submucosal mass extending from $15 \mathrm{~cm}$ to $18 \mathrm{~cm}$ from the incisors with a long stalk, mimicking a large esophageal polyp.

Physical examination of the neck showed no palpable mass. A videoesophagogram showed an oval mass arising from the left side of the cervical esophageal wall with pooling of barium above the mass and a significant delay in passage of contrast beyond the mass (Figure 1, A). Neck computed tomography showed a $2.5-\mathrm{cm}$ mass arising from the left side of the cervical esophageal wall (Figure 1, $B$ and $C$ ). Upper endoscopy showed a left-sided cervical esophageal submucosal mass covered by normal mucosa from 15 to $18 \mathrm{~cm}$ from the incisors, causing a near complete obstruction of the cervical esophagus. There was a long stalk mimicking a large esophageal polyp (Figure 2).

The patient had undergone an esophagogram 2 years before her presentation to us that showed an esophageal mass with no major change in the size relative to that on the recent esophagogram. She had undergone an upper endoscopy at that time, with the result reported as an esophageal polyp.

The patient was taken to the operating room, and the cervical esophagus was exposed through a left neck incision. The recurrent laryngeal nerve was protected. The patient was found to have a palpable round, hard, smoothbordered mass measuring $4 \times 3 \mathrm{~cm}$ underneath the esophageal mucosa inferior to the cricoid. An esophageal myotomy was performed and showed a purple and hypervascular mass. The mass was easily enucleated from the esophageal mucosa (Figure 3, A). The mass originated from the muscular layer. The long-standing effect of the mass on the mucosa had created a $4 \times 4$-cm mucosal diverticulum, which was then imbricated. The muscular layer was closed to cover the mucosa. Intraoperative endoscopy showed a patent lumen and intact mucosa. The patient recovered well and was discharged on postoperative day 1 , tolerating a full liquid diet. She was seen in the clinic on postoperative day 5 . She had an intact voice and was advanced to a soft and then to a regular diet.

The gross pathologic examination showed a wellcircumscribed, tan-purple soft nodule, $2.4 \mathrm{~cm}$ at greatest dimension, with the cut surface spongy and hemorrhagic (Figure 3, B). The microscopic sections showed a benign vascular tumor composed of dilated vessels with thickened walls surrounded by a fibrous capsule. The vascular spaces were filled with red blood cells consistent with a cavernous hemangioma (Figure 3,C).

\section{DISCUSSION}

Esophageal hemangioma is a rare tumor. A review of 106 cases of vascular gastrointestinal tumors showed only 2 cases of esophageal hemangioma. ${ }^{3}$ The most common symptom is dysphagia, followed by hematemesis, melena, and retrosternal pain. The most common location is reported to be the cervical esophagus. ${ }^{4}$ This is a slowgrowing tumor, as shown in our case by long duration of symptoms and the fact that the patient had an esophagogram 2 years before presentation that showed a similar mass and an upper endoscopy that had a reported diagnosis of an esophageal polyp.

In slowly enlarging masses, the progression of dysphagia may be so slow that patients subconsciously adapt and may not report difficulty unless specifically questioned. It is reported that as many as $50 \%$ of elderly people have some dysphagia, which is seldom investigated. ${ }^{5}$ The possibility of mechanical obstruction should nevertheless be considered in all cases. Diagnostic evaluation should include an esophagogram and upper endoscopy and, in selected patients, a computed tomographic scan. In this case, the upper endoscopy showed a mass with a long stalk mimicking an esophageal polyp, but the computed tomographic scan and the videoesophagram showed a mass arising from the esophageal wall. If the therapeutic approach had been based purely on the upper endoscopy, the attempt of endoscopic polypectomy could have resulted in a full-thickness esophageal injury, resulting in a potential leak. This case report emphasizes the importance of using the comprehensive diagnostic modalities, such as esophagography and upper endoscopy in all cases and computed tomographic scan in a selected group of patients for treatment of dysphagia and suspicion for mechanical obstruction. In this case, a comprehensive diagnostic evaluation allowed treatment of a very rare esophageal submucosal mass by simple enucleation without mucosal entry, resulting in resolution of symptoms and a good clinical outcome. 


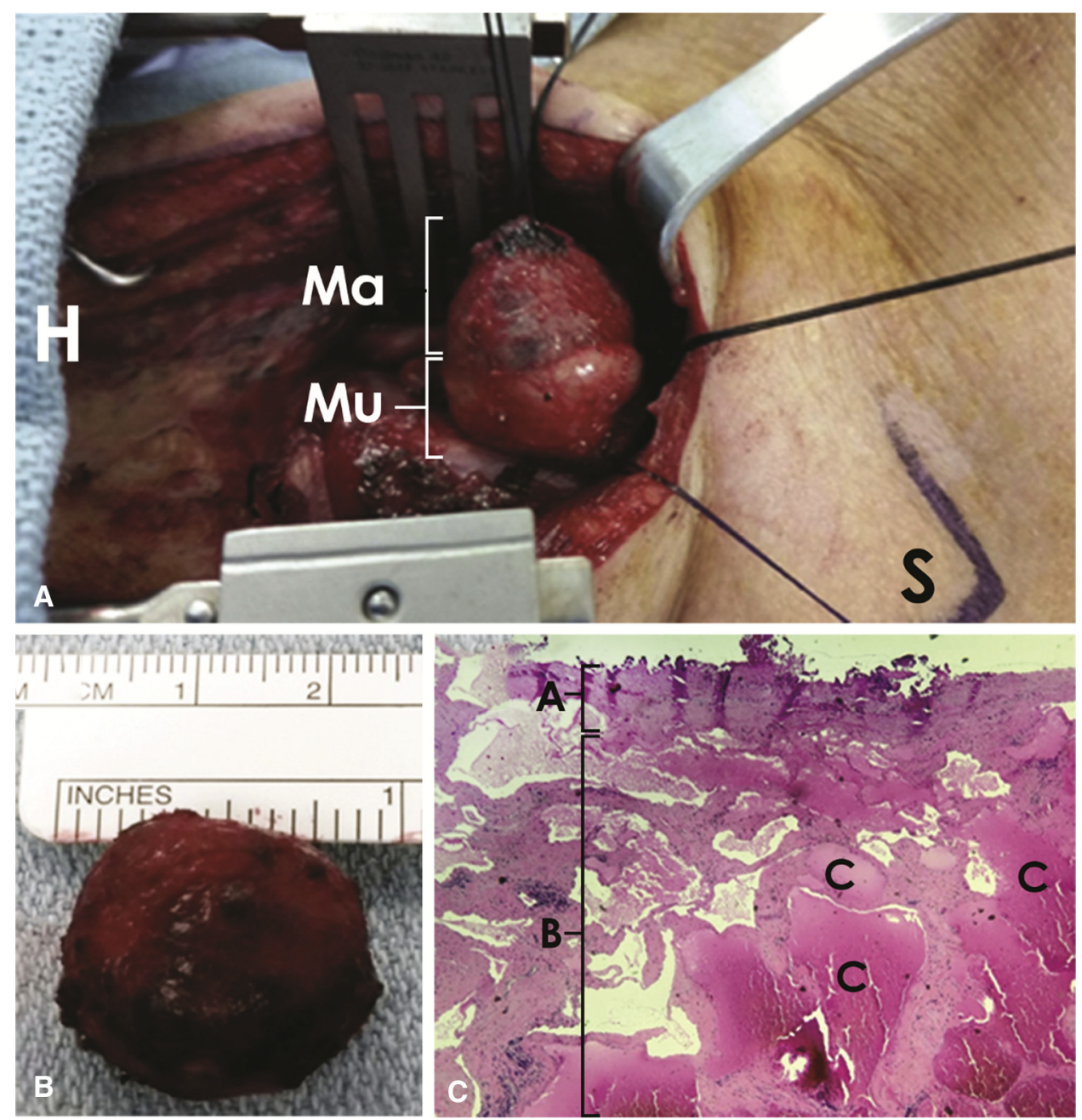

FIGURE 3. A, Intraoperative image of the mass, which is separated from the muscular layer and is still attached to the mucosa. $H$, Head; $M a$, mass; $M u$, esophageal mucosa; $S$, sternal notch. B, A well-circumscribed, tan-purple soft mass, $2.4 \mathrm{~cm}$ in greatest dimension, with the cut surface spongy and hemorrhagic. C, Microscopic section with hematoxylin and eosin stain with magnification $40 \times$ demonstrates a benign vascular tumor composed of dilated vessels with thickened walls $(B)$ surrounded by a fibrous capsule $(A)$. The vascular spaces are filled with red blood cells $(C)$, consistent with cavernous hemangioma. There is no cytologic atypia.

\section{References}

1. Plachta A. Benign tumors of the esophagus: review of literature and report of 99 cases. Am J Gastroenterol. 1962;38:639-52.

2. Araki K, Ohno S, Egashira A, Saeki H, Kawaguchi H, Ikeda Y, et al. Esophageal hemangioma: a case report and review of the literature. Hepatogastroenterology. 1999;46:3148-54.
3. Ghiatas AA, Chopra S, Escobar B, Esola CC, Chintapalli K, Dodd GD III. Esophageal haemangioma. Eur Radiol. 1997;7:1062-3.

4. Wu YC, Liu HP, Liu YH, Hsieh MJ, Lin PJ. Minimal access thoracic surgery for esophageal haemangioma. Ann Thorac Surg. 2001;72:1754-5.

5. Clavé P, Shaker R. Dysphagia: current reality and scope of the problem. Nat Rev Gastroenterol Hepatol. 2015;12:259-70. 